\title{
Light gravitino dark matter: LHC searches and the Hubble tension
}

\author{
Yuchao Gu, ${ }^{1,2, *}$ Maxim Khlopov, ${ }^{3,4,5, \dagger}$ Lei Wu ${ }^{1, \$}$ Jin Min Yang $\odot,{ }^{6,7,8,8}$ and Bin Zhu $\oplus^{2,9, \|}$ \\ ${ }^{1}$ Department of Physics and Institute of Theoretical Physics, Nanjing Normal University, \\ Nanjing 210023, China \\ ${ }^{2}$ School of Physics, Yantai University, Yantai 264005, China \\ ${ }^{3}$ Universit de Paris, CNRS, Astroparticule et Cosmologie, F-75013 Paris, France \\ ${ }^{4}$ Centre for Cosmoparticle Physics Cosmion; National Research Nuclear University MEPHI \\ (Moscow Engineering Physics Institute), Kashirskoe shosse, 31, Moscow 115409, Russia \\ ${ }^{5}$ Institute of Physics, Southern Federal University, Stachki 194, Rostov on Don 344090, Russia \\ ${ }^{6}$ Department of Physics, Tohoku University, Sendai 980-8578, Japan \\ ${ }^{7}$ CAS Key Laboratory of Theoretical Physics, Institute of Theoretical Physics, \\ Chinese Academy of Sciences, Beijing 100190, China \\ ${ }^{8}$ School of Physics, University of Chinese Academy of Sciences, Beijing 100049, China \\ ${ }^{9}$ Department of Physics, Chung-Ang University, Seoul 06974, Korea
}

(Received 18 June 2020; accepted 12 November 2020; published 1 December 2020)

\begin{abstract}
The recent measurements of the cosmological parameter $H_{0}$ from the direct local observations and the inferred value from the cosmic microwave background show approximately $4 \sigma$ discrepancy. This may indicate new physics beyond the standard $\Lambda \mathrm{CDM}$. We investigate the $\mathrm{keV}$ gravitino dark matter that has a small fraction of nonthermal components (e.g., from the late decay of next-to-lightest-supersymmetricparticle bino) under various cosmological constraints. We find such a scenario is highly predictive and can be tested by searching for the dilepton plus missing energy events at the LHC. Besides, we also discuss its implication for Hubble tension, which can be reduced to $3 \sigma$ level marginally.
\end{abstract}

DOI: 10.1103/PhysRevD.102.115005

\section{INTRODUCTION}

The $\Lambda$ CDM model combining cold dark matter (CDM) with a cosmological constant $\Lambda$ is remarkably successful in describing the results of cosmological observations. However, recently, there has been growing tension in the determinations of the Hubble constant, for example, the measurement from Cepheid-calibrated Type Ia Supernova $H_{0}=(74.03 \pm 1.42) \mathrm{km} \mathrm{s}^{-1} \mathrm{Mpc}^{-1}$ [1] shows about $4.4 \sigma$ discrepancy with the inferred value $H_{0}=(67.36 \pm$ 0.54) $\mathrm{km} \mathrm{s}^{-1} \mathrm{Mpc}^{-1}$ [2] from the cosmic microwave background $(\mathrm{CMB})$. Due to the independence of these observations, a simple systematic uncertainty in the data seems impossible to completely solve such a discrepancy [3].

\footnotetext{
*guyc@njnu.edu.cn

khlopov@apc.in2p3.fr

${ }^{*}$ Corresponding author.

leiwu@njnu.edu.cn

§jmyang@itp.ac.cn

Corresponding author. zhubin@mail.nankai.edu.cn

Published by the American Physical Society under the terms of the Creative Commons Attribution 4.0 International license. Further distribution of this work must maintain attribution to the author(s) and the published article's title, journal citation, and DOI. Funded by SCOAP ${ }^{3}$.
}

Therefore, the $H_{0}$ tension may call for new physics beyond the standard $\Lambda \mathrm{CDM}$.

As is known, the cold dark matter can be an explanation for the formation of large-scale structure and galaxies. Despite its success, the predictions made by the CDM deviate from the observational data in small-scale structure, such as core-cusp [4], missing satellite [5], and too big to fail [6] problems. One possible way of solving these problems is to introduce warm dark matter (DM) particles. The freestreaming motion of such warm DM particles reduces power on small scales but keeps the CDM predictions for the formation of large-scale structure. Besides, many models of dark matter in particle physics are not always consisting of the pure CDM. Thus, in conjunction with the Hubble tension, it seems timely to explore the possibilities of departures from the standard CDM model.

Also note that, as a compelling dark matter candidate, the weakly interacting massive particle [7] has been searched for in various (in)direct detections [8] and collider experiments [9]. However, the null results of detection have produced stringent bounds on such interactions, which have motivated us to explore the dark matter at lower masses and/or with different detection signatures (for recent reviews, see, e.g., Refs. [10,11]).

If a DM particle is sufficiently light, it may affect the radiation energy density by mimicking an additional 
neutrino species in the early Universe [12]. During the radiation era, the neutrino energy density $\rho_{\nu}$ in flat geometry is related with the Hubble constant $H(t)$ by

$$
H^{2}(t) \simeq \frac{8 \pi G}{3}\left(\rho_{\gamma}+\rho_{\nu}\right)
$$

where $\rho_{\gamma}$ is the photon energy density. Any process that changes the abundance of neutrinos can alter the expansion rate of the Universe. Interestingly, the Hubble constant and the effective number of neutrino species can have a positive correlation because the nonstandard $N_{\text {eff }}$ can affect the sound horizon, which in turn changes the angular position of the acoustic peaks.

In this paper, we study the light gravitino $(\tilde{G})$ dark matter that is always predicted by locally supersymmetric extensions of the standard model (SM) [13]. Depending on the supersymmetry-breaking mechanisms, the gravitino mass can range from $\mathrm{eV}$ scale up to the scale beyond the $\mathrm{TeV}$ region [14-22]. If the gravitino is the lightest supersymmetric particle (LSP), it can play the role of dark matter particle, which may or may not be in the thermal equilibrium with the hot primordial plasma. When the gravitino dark matter is light enough and nonthermally produced from the late decay of the heavier next-to-LSP (NLSP), it can contribute to the radiation density by mimicking an extra neutrino species. Therefore, such a light gravitino dark matter may reduce the Hubble tension. More interestingly, our framework is highly predictive and can be tested by the LHC experiment.

\section{LIGHT GRAVITINO DARK MATTER}

The gravitino is present in the gauge theory of local supersymmetry. It is the spin- $3 / 2$ superpartner of the graviton. The gravitino interactions are determined by supergravity and by the minimal supersymmetric standard model parameters and are suppressed by the Planck mass. The gravitino mass is obtained via the super-Higgs mechanism [23] and strongly depends on the supersymmetry (SUSY-) breaking schemes. In the gauge mediated supersymmetrybreaking (GMSB) models, the gravitino is usually the LSP and has a mass in the range of $1 \mathrm{eV} \lesssim m_{3 / 2} \lesssim 1 \mathrm{GeV}$ [24]. However, this light gravitino dark matter may lead to some cosmological problems [25-31]. For example, if the gravitino was thermalized in the early Universe, its mass $m_{3 / 2}$ should be less than approximately $1 \mathrm{KeV}$ to avoid overclosing the Universe. Otherwise, a low reheating temperature of inflation $T_{R}$ is required to dilute the gravitino abundance and thus fails to explain the baryon asymmetry by the thermal leptogenesis.

On the other hand, the messenger particles are always predicted by the GMSB models, the superpotential of which is usually given by

$$
W=S \Phi_{M} \bar{\Phi}_{M}+\Delta W\left(S, Z_{i}\right)
$$

where $S$ and $Z_{i}$ are, respectively, the spurion left chiral superfield and the secluded sector fields and $\Phi_{M}$ and $\bar{\Phi}_{M}$ are the messenger left chiral superfields which are charged under the SM gauge group and transmit the SUSY-breaking effect to the visible sector in terms of gauge interaction at the loop level. In the minimal version of the GMSB, the messenger number is conserved so that the lightest messenger particle would easily overclose the Universe, unless it can be diluted to a very low abundance or has a tens of $\mathrm{TeV}$ mass. However, it should be noted that the lightest messenger can have interactions with the SM particles and sparticles by introducing additional messenger-matter interactions or gauge interaction [32-34]. Then, the late decay of the lightest messenger to visible sector particles can produce a substantial amount of entropy and will dilute the light gravitino relic density to the observed value in the present Universe. The dilution factor arising from the messenger decay can be parametrized by

$$
D_{m}=\frac{4 / 3 M_{m} Y_{m}}{\left(90 / g_{*} \pi^{2}\right)^{1 / 4} \sqrt{\Gamma_{m} M_{P}}},
$$

where $Y_{m}$ is the yield of lightest messenger, $M_{m}$ is the mass of messenger, $\Gamma_{m}$ is the messenger decay width, and $g_{*}$ denotes the number of relativisitic degrees of freedom at the temperature of the lightest messenger decay.

Given that the gravitino couplings are extremely weak, the preexisting gravitino can be in or out of the thermal equilibrium in the early Universe. The freeze-out temperature of the gravitino $T_{f}^{3 / 2}$ is given by

$T_{f}^{3 / 2} \approx 0.66 \mathrm{TeV}\left(\frac{g_{*}}{100}\right)^{1 / 2}\left(\frac{m_{3 / 2}}{10 \mathrm{keV}}\right)^{2}\left(\frac{1 \mathrm{TeV}}{m_{\tilde{g}}}\right)^{2}$,

where $g^{*}$ is the effective degrees of freedom of relativistic particles at the gravitino freeze-out temperature and has the value in the range of 90-140 [35]. $m_{\tilde{g}}$ is the mass of gluino and should be heavier than $1 \mathrm{TeV}$ according to the current LHC limits. From Eq. (4), it can be seen that a keV gravitino corresponds to a low freeze-out temperature $T_{f}^{3 / 2} \sim 10 \mathrm{GeV}$. On the other hand, thanks to the messenger dilution effect, the reheating temperature $T_{R}$ can be as high as approximately $10^{9} \mathrm{GeV}$ for the thermal leptogenesis. This indicates that such a light gravitino dark matter in the GMSB should be thermalized in the early Universe, and its relic density can be calculated by

$$
\Omega_{3 / 2}^{\mathrm{TP}} h^{2}=1.14\left(\frac{g_{*}}{100}\right)^{-1}\left(\frac{m_{3 / 2}}{\mathrm{keV}}\right) .
$$

Note that the gravitino can also be nonthermally generated via the late decay of the NLSP, for example, the radiative 
decay of bino, $\tilde{B} \rightarrow \tilde{G} \gamma$ [36-38]. As stated above, such a nonthermal gravitino dark matter may be a solution to the Hubble constant problem. The nonthermal relic density of the gravitino is given by

$$
\begin{aligned}
\Omega_{3 / 2}^{\mathrm{NTP}} h^{2} & =m_{3 / 2} Y_{\tilde{B}}\left(T_{0}\right) s\left(T_{0}\right) h^{2} / \rho_{c} \\
& =\frac{m_{3 / 2}}{m_{\tilde{B}}} \Omega_{\tilde{B}} h^{2},
\end{aligned}
$$

with

$$
\begin{aligned}
\Omega_{\tilde{B}} h^{2}= & 0.0013\left(\frac{m_{\tilde{\ell}_{R}}}{100 \mathrm{GeV}}\right)^{2} \frac{(1+R)^{4}}{R\left(1+R^{2}\right)} \\
& \times\left(1+0.07 \log \frac{\sqrt{R} \times 100 \mathrm{GeV}}{m_{\tilde{\ell}_{R}}}\right),
\end{aligned}
$$

where the mass ratio $R \equiv m_{\tilde{B}^{2}}^{2} / m_{\tilde{\ell}_{R}}^{2}$. Given the strong LHC bounds on the squarks and gluinos, we only include the contributions of the right-handed sleptons to the relic abundance of bino NLSP in Eq. (7) [39]. For simplicity, we assume $m_{\tilde{\ell}_{R}}$ as a common mass parameter of the three generation right-handed sleptons. It should be mentioned that only the first two generations of sleptons are included in Eq. (7) when $m_{\tilde{B}}$ is less than $m_{\tau}$.

Since the decay width of the lightest messenger is much smaller than the gravitino freeze-out temperature $T_{f}^{3 / 2}$, the messenger decay can dilute the thermally produced gravitinos. Besides, the freeze-out temperature of the bino NLSP is usually approximately $m_{\tilde{B}} / 20$. If the bino mass is around $1 \mathrm{GeV}$, it can still freeze out before the messenger decay and then be diluted by the entropy production. It should be noted that the nonthermally produced gravitinos from the bino late decay will not be further diluted as long as the bino decay is sufficiently delayed. Therefore, the final gravitino abundance can be calculated by

$$
\Omega_{3 / 2} h^{2}=\frac{1}{D_{m}}\left(\Omega_{3 / 2}^{\mathrm{TP}} h^{2}+\Omega_{3 / 2}^{\mathrm{NTP}} h^{2}\right) .
$$

In our study, we require that the gravitinos solely compose the dark matter and satisfy the observed relic density within the $3 \sigma$ range, $0.075<\Omega_{3 / 2} h^{2}<0.126$ [40].

Another benefit of the messenger decay in our scenario is that the entropy production can cool down the velocity of the thermally produced gravitino dark matter. For example, when a particle with mass $m$ freezes out from the primordial plasma relativistically, it has a present-day velocity $\left\langle v_{3 / 2}^{0}\right\rangle \approx 0.023 \mathrm{~km} \mathrm{~s}^{-1}\left(g_{*}\left(T_{\mathrm{dec}}\right) / 100\right)^{-1 / 3}(m / 1 \mathrm{keV})^{-1}$, which will be reduced to approximately $\left\langle v_{3 / 2}^{0}\right\rangle / D_{m}^{1 / 3}$. Depending on the dilution factor, the thermally produced gravitino may become nonrelativistic, even if its mass is less than approximately $10 \mathrm{keV}$, whereas, the nonthermally produced gravitino that inherits the kinetic energy from the bino decay can be still relativistic. Because of the vague limits between hot, warm, and cold dark matter, we identify the thermal gravitino dark matter as the CDM when $\left\langle v_{3 / 2}\right\rangle<0.1\left\langle v_{3 / 2}^{0}\right\rangle$ in the following calculations.

\section{CONSTRAINTS}

The gravitino dark matter from the late decay of the bino can be nearly relativistic and thus produce an extra radiation density $\rho_{R}^{\text {extra }}=f \times \rho_{3 / 2} \times\left(\gamma_{3 / 2}-1\right)$ in the early Universe, where $f=\Omega_{3 / 2}^{\mathrm{NTP}} h^{2} /\left(\Omega_{3 / 2}^{\mathrm{TP}} h^{2}+\Omega_{3 / 2}^{\mathrm{NTP}} h^{2}\right)$ is the fraction of the nonthermal gravitino density in the total gravitino production and $\gamma_{3 / 2}$ is the boost factor of the gravitino from the bino decay. At the matter-radiation equality, the energy density per neutrino species is approximately equal to $16 \%$ of the energy density of CDM. This implies that the nonthermal gravitino dark matter that has a kinetic energy equivalent to 1.16 can be regarded as an additional neutrino species. Therefore, the resulting effective neutrino species $\Delta N_{\text {eff }}$ can be given by [12]

$$
\Delta N_{\mathrm{eff}}=f \times\left(\gamma_{3 / 2}-1\right) / 0.16
$$

with

$\gamma_{3 / 2}(a) \simeq 1+\frac{1}{2}\left(\frac{a_{\tau}}{a}\right)^{2} \cdot\left[\left(\frac{m_{\tilde{B}}}{2 m_{3 / 2}}+\frac{m_{3 / 2}}{2 m_{\tilde{B}}}\right)^{2}-1\right]$,

where $a_{\tau}$ is the scale factor at the time of bino decay. In Ref. [41], a comprehensive investigation of the CMB data and direct measurements shows a positive correlation between $N_{\text {eff }}$ and $H_{0}$. For example, when $0.29<$ $\Delta N_{\text {eff }}<0.85$, the Hubble constant can reach $H_{0}=$ $74.03 \mathrm{~km} \mathrm{~s}^{-1} \mathrm{Mpc}^{-1}$. Similar results are also given in Ref. [42]. Thus, increasing the effective number of neutrino species may provide an avenue to ameliorate the Hubble tension. However, it should be noted that the extra relativistic degree of freedom is constrained by the Planck data and baryon acoustic oscillation data, which indicates $N_{\text {eff }}=2.99 \pm 0.17$ [2]. Comparing with the prediction $N_{\text {eff }}=3.046[43,44]$ from the SM with three generations of fermions, this produces an upper bound $\Delta N_{\text {eff }}<0.29$ at 95\% C.L.. We will include this constraint in our following numerical calculations.

On the other hand, we should consider that the nonthermal gravitino will affect the growth of the structure due to its large free-streaming length. The free-streaming starts at the bino decay time and finishes at matter-radiation equality, which is given by

$$
\begin{aligned}
\lambda_{\mathrm{FS}}= & \int_{\tau}^{t_{\mathrm{eq}}} \frac{v_{3 / 2}(t)}{a(t)} d t \\
\simeq & 0.6 \mathrm{Mpc} \times\left(\frac{m_{\tilde{B}}}{10 m_{3 / 2}}\right)\left(\frac{\tau}{10^{4} \mathrm{sec}}\right)^{1 / 2} \\
& \times\left[1+0.1 \log \left(\frac{10 m_{3 / 2}}{m_{\tilde{B}}}\left(\frac{10^{4} \mathrm{sec}}{\tau}\right)^{1 / 2}\right)\right] .
\end{aligned}
$$


If the free-streaming distance that the gravitino propagates is larger than approximately Mpc set by the Lyman-alpha forest [45], it roughly cannot form the observed large-scale structure, which in turn puts a constraint on the nonthemral gravitino dark matter. By fitting the CMB data $[2,46]$, the large-scale structure observations [45], and cosmological simulations [47], it is found that the fraction of the nonthermal gravitino dark matter has to be very small. To suppress such a contribution, one can require the distortion on the linear matter power spectra $\exp (-4.9 f)>$ 0.95 [48,49], which corresponds to $f<0.01$.

Besides, the late decay of bino via the process $\tilde{B} \rightarrow \tilde{G} \gamma$ may affect the big bang nucleosynthesis (BBN) [50], whose life-time in the limit of $m_{\tilde{B}} \gg m_{\tilde{G}}$ is approximately given by

$$
\tau_{\tilde{B}} \simeq \frac{48 \pi M_{P}^{2}}{\cos ^{2} \theta_{W}}\left(\frac{m_{3 / 2}^{2}}{m_{\tilde{B}}^{5}}\right) .
$$

The photons from the bino decay may induce electromagnetic showers through their scattering off the background photons and electrons [51,52]. The energetic photon in the shower can destroy the light elements such as D and ${ }^{4} \mathrm{He}$. The photodissociation of ${ }^{4} \mathrm{He}$ happens at the cosmic time of greater than or approximately equal to $10^{6} \mathrm{~s}$, while photodissociation of $\mathrm{D}$ will be important at higher temperature because of the smallness of its binding energy, which corresponds to a long-lived particle with a lifetime longer than $10^{4} \mathrm{~s}[53,54]$. Thus, we require the lifetime of our late decaying bino to be shorter than $10^{4} \mathrm{~s}$ to avoid the BBN constraints.

\section{NUMERICAL RESULTS AND DISCUSSIONS}

Next, we perform a numerical study to explore the allowed parameter space of our scenario. There are only four relevant input parameters in our scenario: $m_{\tilde{B}}, m_{\tilde{G}}$, $m_{\tilde{\ell}_{R}}$, and $D_{m}$. We consider all the constraints stated above.

In Fig. 1, we present the results of the lifetime of bino $(\tau)$, the mass ratio $m_{\tilde{B}} / m_{\tilde{G}}$, the nonthermal gravitino DM fraction $f$, and the dilution factor $D_{m}$ for the samples allowed by the experimental constraints. It can be seen that there is a strong correlation between these quantities. The lifetime of bino deceases as the mass ratio $m_{\tilde{B}} / m_{\tilde{G}}$ becomes large, which can be much smaller than the BBN bound. The dilution factor is required to be in the range of $29<D_{m}<266$. The nonthermal gravitino DM fraction $f$ can be suppressed to $\mathcal{O}\left(10^{-3}\right)$. When $m_{\tilde{B}}$ is fixed, a light gravitino will lead to a small thermal relic density of the gravitino DM, while a heavy gravitino will need a large dilution factor to reduce the thermal relic density. Both cases can result in a large value of $f$. On the other hand, for a given slepton mass, a heavy $m_{\tilde{B}}$ will increase the relic density of nonthermal gravitino DM and thus enhance the value of $f$.

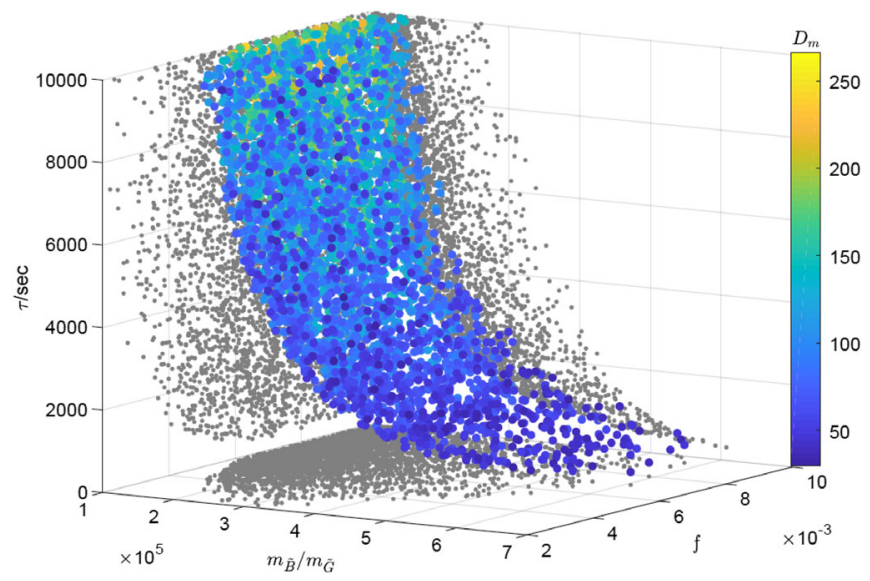

FIG. 1. Samples satisfying the DM relic density $(0.075<$ $\left.\Omega h^{2}<0.126\right)$, the BBN constraint $\left(\tau_{\tilde{B}}<10^{4} \mathrm{~s}\right)$, and other cosmological observations $\left(f<0.01\right.$ and $\left.N_{\text {eff }}<0.29\right)$. The color map denotes the values of dilution factor.

We comment on the possible realization of a large dilution factor $D_{m}$ in Fig. 1. For example, in the general gauge mediation, the messenger sector can be $5 \oplus \overline{5}$ representation under $S U(5)$. The interaction between messenger and matter fields in minimal Kahler potential can be written as [33]

$$
\delta K=\lambda \Phi_{m} \overline{5},
$$

where $\lambda$ is an $\mathcal{O}(1)$ parameter from the naturalness criteria and $\overline{5}$ stands for matter fields in the SM. In terms of Kahler transformation, this interaction can be reinterpreted as additional interaction in superpotential,

$$
\delta W=\lambda m_{3 / 2} \Phi_{m} \overline{5} .
$$

Assuming that only gauge interaction plays a crucial role, the decay width of messenger is approximately given by [55]

$$
\Gamma_{m}=\frac{g^{2}}{16 \pi}\left(\frac{m_{3 / 2}}{\sqrt{2} M_{m}}\right)^{2} M_{m}
$$

Here, it can be seen that the appearance of the small factor $\left(m_{3 / 2} / M_{m}\right)^{2}$ will naturally lead to a tiny decay width of the lightest messenger. When $M_{m}$ is about $\mathcal{O}\left(10^{8}\right) \mathrm{GeV}$, the corresponding yield $Y_{m}$ is around $\mathcal{O}\left(10^{-9}\right)$, and the decay width of the lightest messenger is about $\mathcal{O}\left(10^{-22}\right) \mathrm{GeV}$. Then, one can have a dilution factor of $\mathcal{O}(10)-\mathcal{O}\left(10^{2}\right)$. As an example, we present a benchmark point in Table I.

TABLE I. A benchmark point for the dilution factor $D_{m}$.

\begin{tabular}{ccccc}
\hline \hline$M_{m}(\mathrm{GeV})$ & $m_{32}(\mathrm{keV})$ & $Y_{m}$ & $\Gamma_{m}(\mathrm{GeV})$ & $D_{m}$ \\
\hline $3 \times 10^{8}$ & 5 & $7.1 \times 10^{-9}$ & $3.5 \times 10^{-22}$ & 100 \\
\hline \hline
\end{tabular}




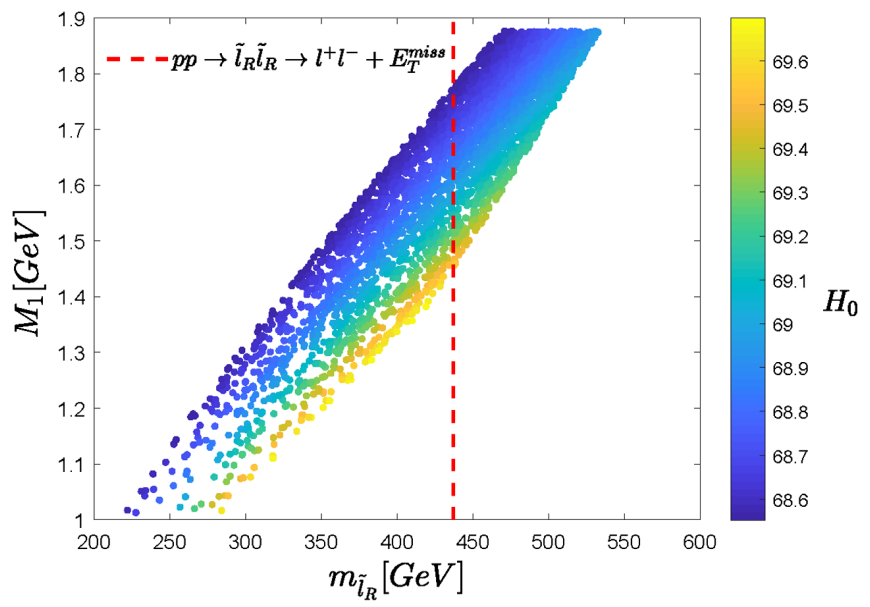

FIG. 2. Same as Fig. 1, but projected on the plane of $m_{\tilde{B}}$ vs $m_{\tilde{\ell}_{R}}$. The color map denotes the values of Hubble constant. The dashed line corresponds to the $95 \%$ C.L. exclusion limits from the search for the slepton pair events at the $13 \mathrm{TeV}$ LHC with a luminosity of $\mathcal{L}=139 \mathrm{fb}^{-1}[56]$.

In Fig. 2, we show the allowed samples on the plane of $m_{\tilde{B}}$ vs $m_{\tilde{\ell}_{R}}$. Following Ref. [42], we calculate the corresponding values of Hubble constant for these samples and present their results as well. It can be seen that the Hubble constant can be mostly enhanced to about $H_{0}=69.69$, which reduces the tension to around $3 \sigma$ level. It should be noted that the required effective neutrino number $\Delta N_{\text {eff }}$ and the large-scale observation produces a lower and upper limit on the fraction $f$, respectively. These lead to the bounds on the slepton mass, since the nonthermal gravitino DM relic density depends on the abundance of bino. From Fig. 2, we can see that the slepton mass $m_{\tilde{\ell}}$ has to be less than about $520 \mathrm{GeV}$. Such a light slepton can be produced in pair through the Drell-Yan process $p p \rightarrow \tilde{\ell}^{+} \tilde{\ell}^{-}$at the LHC. Because of the small mass splitting between the bino and gravitino, the photon from the bino decay will be too soft to be observed by the detectors. Therefore, such a slepton pair production process will give the dilepton plus missing energy signature at the LHC. In Fig. 2, we present the current LHC bounds of searching for selectron/smuon pair production and find that the slepton with the mass lighter than about $440 \mathrm{GeV}$ has been excluded. We can expect that the rest of parameter space can be fully probed by the high luminosity LHC.

\section{CONCLUSIONS}

In this paper, we studied the $\mathrm{keV}$ gravitino dark matter with a small fraction of nonthermal relic density in the gauge mediation supersymmetry breaking. Thanks to the messenger decay, the gravitino abundance can be diluted to the observed value and also make the thermally produced gravitino still cold to satisfy the large-scale structure observations. We found that such a scenario can be tested by searching for slepton pair production at the LHC. Besides, since the nonthermal gravitino from the bino decay can mimic additional relativistic species, the expansion rate of the Universe could be altered in the early Universe. However, because of the current strong constraint on the effective neutrino number $\Delta N_{\text {eff }}$, the Hubble tension can only be reduced to about $3 \sigma$ level at best.

\section{ACKNOWLEDGMENTS}

We appreciate Feng Luo for his collaboration on early stage and thank Xin Zhang for his helpful discussions. This work is supported by the National Natural Science Foundation of China (NNSFC) under Grants No. 117050934, No. 11847208, No. 11875179, No. 11805161, No. 11675242, No. 11821505, and No. 11851303; by Jiangsu Specially Appointed Professor Program; by Peng-Huan-Wu Theoretical Physics Innovation Center (Grant No. 11847612); by the CAS Center for Excellence in Particle Physics (CCEPP); by the CAS Key Research Program of Frontier Sciences; and by a Key R\&D Program of Ministry of Science and Technology under Grant No. 2017YFA0402204 and Natural Science Foundation of Shandong Province under Grant No. ZR2018QA007. B.Z. is also supported by the Basic Science Research Program through the National Research Foundation of Korea (NRF) funded by the Ministry of Education, Science and Technology (Grant No. NRF-2019R1A2C2003738) and by the Korea Research Fellowship Program through the NRF funded by the Ministry of Science and ICT (Grant No. 2019H1D3A1A01070937). The work by M. K. was supported by the Ministry of Science and Higher Education of the Russian Federation as part of the Program for Improving the Competitiveness of the MEPhI (Project No. 02.a03.21.0005) and Project "Fundamental problems of cosmic rays and dark matter," Project No. 07232020-0040. 
[1] A. G. Riess, S. Casertano, W. Yuan, L. M. Macri, and D. Scolnic, Astrophys. J. 876, 85 (2019).

[2] N. Aghanim et al. (Planck Collaboration), Astron. Astrophys. 641, A6 (2020).

[3] L. Verde, T. Treu, and A. Riess, Nat. Astron. 3, 891 (2019).

[4] M. G. Walker and J. Penarrubia, Astrophys. J. 742, 20 (2011).

[5] A. A. Klypin, A. V. Kravtsov, O. Valenzuela, and F. Prada, Astrophys. J. 522, 82 (1999).

[6] M. Boylan-Kolchin, J. S. Bullock, and M. Kaplinghat, Mon. Not. R. Astron. Soc. 415, L40 (2011).

[7] B. W. Lee and S. Weinberg, Phys. Rev. Lett. 39, 165 (1977).

[8] G. Jungman, M. Kamionkowski, and K. Griest, Phys. Rep. 267, 195 (1996).

[9] O. Buchmueller, C. Doglioni, and L. T. Wang, Nat. Phys. 13, 217 (2017).

[10] S. Knapen, T. Lin, and K. M. Zurek, Phys. Rev. D 96, 115021 (2017).

[11] G. Bertone and T. M. P. Tait, Nature (London) 562, 51 (2018).

[12] D. Hooper, F. S. Queiroz, and N. Y. Gnedin, Phys. Rev. D 85, 063513 (2012).

[13] J. Wess and J. Bagger, Supersymmetry and Supergravity (Princeton University Press, Princeton, NJ, 1992).

[14] H. Pagels and J. R. Primack, Phys. Rev. Lett. 48, 223 (1982).

[15] S. Weinberg, Phys. Rev. Lett. 48, 1303 (1982).

[16] M. Khlopov and A. D. Linde, Phys. Lett. B 138, 265 (1984).

[17] M. Dine, A. E. Nelson, and Y. Shirman, Phys. Rev. D 51, 1362 (1995).

[18] G. Giudice and R. Rattazzi, Phys. Rep. 322, 419 (1999).

[19] L. Randall and R. Sundrum, Nucl. Phys. B557, 79 (1999).

[20] J. R. Ellis, K. A. Olive, Y. Santoso, and V. C. Spanos, Phys. Lett. B 588, 7 (2004).

[21] W. Buchmuller, K. Hamaguchi, and J. Kersten, Phys. Lett. B 632, 366 (2006).

[22] E. Dudas, Y. Mambrini, and K. Olive, Phys. Rev. Lett. 119, 051801 (2017).

[23] E. Cremmer, S. Ferrara, L. Girardello, and A. Van Proeyen, Nucl. Phys. B212, 413 (1983).

[24] G. F. Giudice, M. A. Luty, H. Murayama, and R. Rattazzi, J. High Energy Phys. 12 (1998) 027.

[25] T. Moroi, H. Murayama, and M. Yamaguchi, Phys. Lett. B 303, 289 (1993).

[26] T. Asaka, K. Hamaguchi, and K. Suzuki, Phys. Lett. B 490, 136 (2000).

[27] M. Bolz, A. Brandenburg, and W. Buchmuller, Nucl. Phys. B606, 518 (2001); B790, 336(E) (2008).

[28] L. Roszkowski, R. Ruiz de Austri, and K.-Y. Choi, J. High Energy Phys. 08 (2005) 080.
[29] D. G. Cerdeno, K.-Y. Choi, K. Jedamzik, L. Roszkowski, and R. Ruiz de Austri, J. Cosmol. Astropart. Phys. 06 (2006) 005.

[30] J. Pradler and F. D. Steffen, Phys. Rev. D 75, 023509 (2007).

[31] J. Pradler and F. D. Steffen, Phys. Lett. B 648, 224 (2007).

[32] E. A. Baltz and H. Murayama, J. High Energy Phys. 05 (2003) 067.

[33] M. Fujii and T. Yanagida, Phys. Lett. B 549, 273 (2002).

[34] K. Jedamzik, M. Lemoine, and G. Moultaka, Phys. Rev. D 73, 043514 (2006).

[35] E. Pierpaoli, S. Borgani, A. Masiero, and M. Yamaguchi, Phys. Rev. D 57, 2089 (1998).

[36] J. L. Feng, A. Rajaraman, and F. Takayama, Phys. Rev. Lett. 91, 011302 (2003).

[37] J. L. Feng, A. Rajaraman, and F. Takayama, Phys. Rev. D 68, 063504 (2003).

[38] J. L. Feng, S. Su, and F. Takayama, Phys. Rev. D 70, 075019 (2004).

[39] N. Arkani-Hamed, A. Delgado, and G. Giudice, Nucl. Phys. B741, 108 (2006).

[40] P. Ade et al. (Planck Collaboration), Astron. Astrophys. 594, A13 (2016).

[41] J. L. Bernal, L. Verde, and A. G. Riess, J. Cosmol. Astropart. Phys. 10 (2016) 019.

[42] S. Vagnozzi, Phys. Rev. D 102, 023518 (2020).

[43] G. Mangano, G. Miele, S. Pastor, T. Pinto, O. Pisanti, and P. D. Serpico, Nucl. Phys. B729, 221 (2005).

[44] K. Akita and M. Yamaguchi, J. Cosmol. Astropart. Phys. 08 (2020) 012.

[45] M. Viel, M. G. Haehnelt, and V. Springel, J. Cosmol. Astropart. Phys. 06 (2010) 015.

[46] C. Fidler, A. Kleinjohann, T. Tram, C. Rampf, and K. Koyama, J. Cosmol. Astropart. Phys. 01 (2019) 025.

[47] J. Liu, S. Bird, J. M. Z. Matilla, J. C. Hill, Z. Haiman, M. S. Madhavacheril, A. Petri, and D. N. Spergel, J. Cosmol. Astropart. Phys. 03 (2018) 049.

[48] C.-P. Ma, Astrophys. J. 471, 13 (1996).

[49] D. J. Eisenstein and W. Hu, Astrophys. J. 511, 5 (1999).

[50] J.-M. Yang, M. S. Turner, G. Steigman, D. Schramm, and K. A. Olive, Astrophys. J. 281, 493 (1984).

[51] R. H. Cyburt, J. Ellis, B. D. Fields, F. Luo, K. A. Olive, and V. C. Spanos, J. Cosmol. Astropart. Phys. 10 (2009) 021.

[52] M. Kawasaki, K. Kohri, T. Moroi, and Y. Takaesu, Phys. Rev. D 97, 023502 (2018).

[53] E. Holtmann, M. Kawasaki, K. Kohri, and T. Moroi, Phys. Rev. D 60, 023506 (1999).

[54] M. Kawasaki, K. Kohri, and T. Moroi, Phys. Rev. D 63, 103502 (2001).

[55] F. Staub, W. Porod, and J. Niemeyer, J. High Energy Phys. 01 (2010) 058.

[56] G. Aad et al. (ATLAS Collaboration), Eur. Phys. J. C 80, 123 (2020). 